\title{
Analysis of the expression level and methylation of tumor protein p53, phosphatase and tensin homolog and mutS homolog 2 in N-methyl-N-nitrosourea-induced thymic lymphoma in C57BL/6 mice
}

\author{
XUEYUN HUO, ZHENKUN LI, SHUANGYUE ZHANG, CHANGLONG LI, \\ MENG GUO, JING LU, JIANYI LV, XIAOYAN DU and ZHENWEN CHEN
}

\begin{abstract}
Department of Medical Genetics, School of Basic Medical Science, Capital Medical University, Beijing 100069, P.R. China
\end{abstract}
Received January 4, 2016; Accepted May 23, 2017

DOI: $10.3892 / \mathrm{ol} .2017 .6721$

\begin{abstract}
Tumorigenesis is often caused by somatic mutation or epigenetic changes in genes that regulate aspects of cell death, proliferation and survival. Although the functions of multiple tumor suppressor genes have been well studied in isolation, how these genes cooperate during the progression of a single tumor remains unclear in numerous cases. The present study used N-methyl-N-nitrosourea (MNU), one of the most potent mutagenic nitrosourea compounds, to induce thymic lymphoma in C57BL/6J mice. Subsequently, the protein expression levels of phosphatase and tensin homolog (PTEN), transformation protein 53 and mutS homolog 2 (MSH2) were evaluated concomitantly in the thymus, liver, kidney and spleen of MNU-treated mice by western blotting. To determine whether changes in expression level were due to aberrant epigenetic regulation, the present study further examined the methylation status of each gene by MassARRAY analysis. During the tumorigenesis process of an MNU-induced single thymic lymphoma, the expression level of PTEN was revealed to be reduced in thymic lymphoma samples but not in normal or non-tumor thymus tissue samples. Furthermore, a marked reduction of P53 expression levels were demonstrated in thymic lymphomas and spleens with a metastatic tumor. Conversely, MSH2 upregulation was identified only in liver, kidney, and spleen samples that were infiltrated by thymic lymphoma cells. Furthermore, the present study revealed that a number of 5'-C-phosphate-G-3' sites located in the promoter of aberrantly
\end{abstract}

Correspondence to: Professor Zhenwen Chen or Professor Xiaoyan Du, Department of Medical Genetics, School of Basic Medical Science, Capital Medical University, 10 Xitoutiao Youwai Street, Beijing 100069, P.R. China

E-mail: czwen@ccmu.edu.cn

E-mail: duduyan@ccmu.edu.cn

Key words: transformation protein 53, phosphatase and tensin homolog, mutS homolog 2, N-methyl-N-nitrosourea, thymic lymphoma, DNA methylation, C57BL/6J mice expressed genes had significantly altered methylation statuses. These results improve the understanding of the course of mutagen-induced cancer, and highlight that epigenetic regulation may serve an important function in cancer.

\section{Introduction}

Tumor development is a complex process that involves genetic and epigenetic abnormalities. These alterations may contribute to the inactivation of tumor suppressor genes and eventually induce tumorigenesis (1).

Phosphatase and tensin homolog (PTEN) is a lipid phosphatase that negatively regulates the phosphoinositide 3 -kinase/protein kinase $\mathrm{B} /$ mechanistic target of rapamycin signaling axis and thereby inhibits cell proliferation and metabolism (2). Tumor protein 53 (P53) is a DNA sequence-specific transcription factor that is able to selectively activate genes involved in cell cycle arrest, apoptosis and senescence in response to diverse cellular stresses (3). Accordingly, PTEN and P53 are key suppressors in neoplastic progression and are inactivated via gene mutation $(4,5)$ or aberrant DNA methylation $(6,7)$ in numerous types of malignant tumor.

MutS homolog 2 (MSH2) is an essential component of the DNA mismatch repair (MMR) singling pathway, which is essential for maintenance of genome integrity as it identifies and corrects mismatched nucleotides and triggers homologous recombination during DNA replication (8). Defects in the $\mathrm{MSH} 2$ gene abrogate the MMR response (9). In addition, these engender genome-wide instability and thus increase the risk of malignant transformation (10). Although the three aforementioned tumor suppressor genes have been well-studied separately, the alterations in their expression levels during the progression of a single tumor remain unclear, and may reveal the combined contribution of the three genes to the initiation, progression and maintenance of a single tumor.

Genetic changes, including mutations, have a clear-cut function in tumor progression; however, the mechanistic insights and importance of epigenetic changes in carcinogenesis are comparatively less well understood (11). DNA methylation is the best-known epigenetic modification, and aberrant methylation in the promoter region 5'-C-phosphate-G-3' (CpG) 
islands is associated with gene silencing, as this downregulates transcription $(12,13)$. In human types of cancer, altered methylation patterns epigenetically repress tumor suppressor genes, providing a direct link between epigenetic regulation and cancer (14).

$\mathrm{N}$-methyl-N-nitrosourea (MNU) is one of the most potent mutagenic nitrosourea compounds due to its ability to interact directly with, and alkylate, genomic DNA (15). MNU is able to induce numerous types of mammary tumors, and MNU-induced thymic lymphoma is a well-established animal model (16). In a previous study by our group, a relatively high frequency $(61.3 \%)$ of thymic lymphoma was induced in C57BL/6J mice using MNU (17); however, the complete process of thymic lymphoma induced by MNU in these mice was not determined. In an attempt to describe the process of tumorigenesis more comprehensively, the present study determined the protein expression status of PTEN, transformation protein 53 (TRP53) and MSH2 simultaneously in various tissues of MNU-exposed mice, and further explored the promoter methylation status of each gene by MassARRAY analysis.

\section{Materials and methods}

Ethics statement. All of the experiments and animal procedures were performed in accordance with the Guidelines of Capital Medical University Animal Experiments and Experimental Animals Management Committee (Beijing, China). The protocol was approved by the Animal Experiments and Experimental Animal Welfare Committee of Capital Medical University (permit no. 2011-X-009).

Animal experiments. The MNU-induced thymic lymphoma model in C57BL/6J mice was developed as previously described (17). In brief, 5-6-week-old (15-20 g body weight) C57BL/6J male mice (The Academy of Military Medical Sciences, Beijing, China) were used in the present study. The animals ( 5 mice per cage) were housed in a barrier system and were maintained in controlled conditions: Temperature $\left(23 \pm 2^{\circ} \mathrm{C}\right)$, humidity $(55 \pm 10 \%)$ and lighting $(12 \mathrm{~h}$ light/dark cycle). The mice were allowed access to food and water ad libitum. The mice were allowed to acclimate to the environment for 1 week prior to commencement of the experiments.

MNU (Sigma-Aldrich; Merck KGaA, Darmstadt, Germany) was dissolved in dimethyl sulfoxide (DMSO) and diluted in phosphate buffer (PBS, $\mathrm{pH}$ 7.2) to a concentration of $5 \mathrm{mg} / \mathrm{ml}$ immediately prior to use. A total of 40 mice in the MNU group were administered a single intraperitoneal dose of $90 \mathrm{mg} / \mathrm{kg}$ body weight and 10 mice in the control group were injected with the solvent only (DMSO and PBS) in a corresponding dose of $90 \mathrm{mg} / \mathrm{kg}$ body weight. The animals were euthanized 16 weeks following the initial injection and the liver, kidney, spleen and thymus of each mouse were immediately collected. Thymic lymphomas and the metastatic tumors in the liver, kidney and spleen were identified by morphological and immunohistochemical studies as previously described (17).

Western blot analysis. A total of 9 mice in three groups, including the control group $(n=3)$, tumor group $(n=3$; mice with thymic lymphoma and tumor metastasis to the liver, kidney and spleen) and non-tumor group $(\mathrm{n}=3$; mice with no tumors in the thymus, liver, kidney and spleen) were used to investigate the expression levels of TRP53, PTEN and MSH2. Proteins were extracted from the thymus, livers, spleens and kidneys of each mice using a Protein Extraction kit (CWBio, Beijing, China) and quantified using BCA-Reagents (CWBio). Protein lysates (20 $\mu \mathrm{g} / \mathrm{lane})$ were subjected to SDS-PAGE at $160 \mathrm{~V}$ on a $12 \%$ gel (CWBio) for $1 \mathrm{~h}$, and then transferred to a $0.45-\mu \mathrm{m}$ nitrocellulose filter membrane (buffer: Tris $5.8 \mathrm{~g}$, glycine $2.9 \mathrm{~g}$, SDS $0.376 \mathrm{~g}$, methyl alcohol $200 \mathrm{ml}$ to $1 \mathrm{l}$ ) at $200 \mathrm{~mA}$ for $2 \mathrm{~h}$ using Semi Dry Transfer method (ice-bath). Primary antibodies against P53 (cat no. 2524 s), PTEN (cat no. 9559S) and MSH2 (cat no. 2017S) were diluted to $1: 1,000$. GAPDH antibodies (cat no. $5174 \mathrm{~S}$ ) were diluted to $1: 2,000$. All of the primary antibodies were incubated at $4^{\circ} \mathrm{C}$ for $12 \mathrm{~h}$. The secondary antibodies (goat anti-rabbit IgG conjugated to horseradish peroxidase; cat no. 7074S) were diluted to $1: 5,000$ and incubated at room temperature for $1 \mathrm{~h}$ (all the antibodies were purchased from Cell Signaling Technology, Inc., Danvers, MA, USA). The membranes were washed thoroughly (Tris-buffered saline with Tween) and visualized using enhanced chemiluminescence detection reagents (Thermo Fisher Scientific, Inc., Waltham, MA, USA). Semi-quantitative results were normalized to the housekeeping gene, GAPDH, following scanning to obtain a grayscale image (Gel DocXR System; 1708170EDU; Bio-Rad Laboratories, Inc., Hercules, CA, USA).

DNA methylation analysis. The promoter $\mathrm{CpG}$ islands of Trp53, Pten and Msh2 were identified using CpG Island Searcher (http://cpgislands.usc.edu) between 5,000-1,000 base pairs of the transcriptional start site. Primers for amplifying the upstream $\mathrm{CpG}$ islands were designed using Methyl Primer Express version 1.0 (Applied Biosystems; Thermo Fisher Scientific, Inc.) and Vector NTI Advance 10 (Invitrogen; Thermo Fisher Scientific, Inc.; Table I). The methylation status of the three genes was analyzed in the thymus, liver, kidney and spleen of 26 mice (control=6, tumor=10, non-tumor=10) using MassARRAY Spectrometry, as previously described (18). Briefly, $1 \mu \mathrm{g}$ genomic DNA was isolated using standard phenol-chloroform extraction and ethanol precipitation (anhydrous ethanol, $-20^{\circ} \mathrm{C}$ ) method, and was treated with sodium bisulfate (3.1 M, Sigma-Aldrich; Merck KGaA) for $16 \mathrm{~h}$ at $55^{\circ} \mathrm{C}$. Polymerase chain reaction amplifications were performed to obtain the $\mathrm{CpG}$ island sequences of the three genes as follows: Amplifications were performed in $100 \mu \mathrm{l}$ reaction mixtures containing $5 \mu \mathrm{l}$ bisulphite-treated genomic DNA, $200 \mu \mathrm{M}$ dNTPs, luM primers, $3 \mathrm{mM} \mathrm{MgCl}_{2}, 50 \mathrm{mM}$ KCI, 10 mM Tris-HCI pH 8.3, $0.5 \mu \mathrm{l}$ (2.5 units) AmpliTaq DNA polymerase (Cetus), in a gradient thermal cycler (Bio-Rad Laboratories, Inc.; ALS1296) under the following conditions: $94^{\circ} \mathrm{C} / 2 \mathrm{~min}$ for $1 \mathrm{cycle} ; 94^{\circ} \mathrm{C} / 1 \mathrm{~min}, 50^{\circ} \mathrm{C} / 2 \mathrm{~min}$, $72^{\circ} \mathrm{C} / 3 \mathrm{~min}$ for 5 cycles; $94^{\circ} \mathrm{C} / 0.5 \mathrm{~min}, 50^{\circ} \mathrm{C} / 2 \mathrm{~min}, 72^{\circ} \mathrm{C}$ $/ 1.5 \mathrm{~min}$ for 25 cycles; $72^{\circ} \mathrm{C} / 6 \mathrm{~min}$ for 1 cycle. Subsequently, DNA methylation status was quantitatively analyzed by matrix-assisted laser desorption ionization-time of flight mass spectrometry (18), and methylation data for each CpG site was generated using EpiTyper version 1.0.5 software (Sequenom, San Diego, CA, USA).

Statistical analysis. Statistical analysis was performed using GraphPad Prism version 5.0 (GraphPad Software, 
Table I. Primer information for Trp53, Pten and Msh2.

\begin{tabular}{|c|c|c|c|}
\hline Gene & Primer & $\begin{array}{l}\text { Product } \\
\text { size (bp) }\end{array}$ & $\begin{array}{l}\text { Detectable } \\
\text { CpG sites }\end{array}$ \\
\hline $\operatorname{Trp53}$ & $\begin{array}{l}\text { Forward: 5'-aggaagagagGGTTAGGTTAGGAGGGAGGTTATT-3' } \\
\text { Reverse: 3'cagtaatacgactcactatagggagaaggctCAAAACCCAAAATTCAAACTACAAC-5' }\end{array}$ & 427 & 26 \\
\hline Pten & $\begin{array}{l}\text { Forward: 5'-aggaagagagGGATGTGGTTGTTTGTGTAATTAGT-3' } \\
\text { Reverse: 3'-cagtaatacgactcactatagggagaaggctAATCACAACCAAACTCAATCTTCAA-5' }\end{array}$ & 516 & 22 \\
\hline Msh2 & $\begin{array}{l}\text { Forward: 5'-aggaagagagTTGGGTTAGTAAGAGGTTGTGTAGAA-3' } \\
\text { Reverse: 3'-cagtaatacgactcactatagggagaaggctACCAACACCACTAAAACACAAAAC-5' }\end{array}$ & 478 & 22 \\
\hline
\end{tabular}

Trp53, transformation protein 53; Pten, phosphatase and tensin homolog; Msh2, mutS homolog 2.

Inc., La Jolla, CA, USA). The comparisons between various groups were performed using the $\chi^{2}$ test and one-way analysis of variance followed by Tukey's post-hoc test. $\mathrm{P}<0.05$ was considered to indicate a statistically significant difference.

\section{Results}

Protein expression levels of TRP53, PTEN and MSH2 in various tissues following $M N U$ treatment. In the present study, the protein expression levels of TRP53, PTEN and MSH2 in MNU-induced thymus, liver, spleen and kidney tumor tissues and normal non-exposed tissue samples were determined by western blotting.

It was revealed that MNU treatment reduced the expression level of TRP53 protein in tumor tissues. The TRP53 expression level in thymic lymphoma tissues and non-tumor thymus tissue was significantly lower compared with in normal non-treated thymus tissues $(\mathrm{P}=0.007$ and $\mathrm{P}=0.031$, respectively; Fig. 1; Table II). In the spleen, the expression level of P53 was significantly repressed in the tumor group compared with in the control and non-tumor groups $(\mathrm{P}=0.009$ and $\mathrm{P}=0.008$, respectively). As presented in Fig. 1, all the tumor groups in the thymus, liver, spleen and kidney tissues exhibited a reduced TRP53 expression level compared with the control group and the non-tumor treatment group, particularly in the thymus and spleen. However, TRP53 expression level in the non-tumor groups, except in the thymus, did not affect the development of tumors in the mice although they were also treated with MNU.

The present study demonstrated that PTEN was downregulated in MNU-induced thymic lymphoma tissues relative to the control and non-tumor groups $(\mathrm{P}=0.027$ and $\mathrm{P}=0.01$, respectively). Conversely, PTEN expression levels were not downregulated in tumor or non-tumor groups of the liver, kidney and spleen compared with the control group. In addition, the PTEN expression level in the liver and kidney were slightly increased compared with the control (Fig. 2; Table II).

The expression level of MSH2 revealed distinctive features in the thymus, liver, spleen and kidney tissues. In the thymus tissue, MNU treatment did not induce any significant changes in MSH2 expression levels, including in thymic lymphomas and thymus tissue from the non-tumor group. Of note, the expression levels of MSH2 in the liver, kidney and spleen in the three groups were similar to each other. An elevation in MSH2 expression levels was observed in tumor-bearing tissues compared with in control groups $(\mathrm{P}<0.001, \mathrm{P}=0.003$ and $\mathrm{P}=0.012$ for the liver, kidney and spleen, respectively) and non-tumor groups $(\mathrm{P}<0.000$ and $\mathrm{P}=0.002$ for the liver and kidney, respectively; Fig. 3; Table II).

Trp53 methylation status. Subsequently, MassARRAY analysis was performed to detect the methylation status of the genes for which protein expression level was markedly altered.

In the thymus, among the 26 detected $\mathrm{CpG}$ units in the Trp53 promoter, 3 (CpG-2.3, CpG-12.13 and CpG-24; 3/26, $11.5 \%$ ) were differently methylated between the control, tumor and non-tumor groups (Fig. 4; Table III). The methylation of CpG-2.3 in the non-tumor group was significantly lower compared with in the control group $(\mathrm{P}=0.033)$; however, there was no statistical difference between the tumor group and the other two groups (control and non-tumor) regarding methylation status. The methylation of CpG-12.13 in the tumor group was notably higher compared with in the control group $(\mathrm{P}=0.008)$ and non-tumor group $(\mathrm{P}=0.044)$; however, there was no significant difference in methylation status between the control and non-tumor groups. The methylation of CpG-24 in the tumor group was lower compared with in the non-tumor group $(\mathrm{P}=0.047)$, whereas no statistical difference in methylation status was revealed between controls and the other two groups. In addition, CpG-24 methylation was increased in the tumor group compared with in the control group $\mathrm{P}=0.07$; Fig. 4; Table III).

In the spleen, there were no statistically significant differences in the methylation of $\mathrm{CpG}$ sites between the control, tumor and non-tumor groups; however, the methylation of CpG-5 tended towards a decrease in the tumor group and non-tumor group when compared with the control group $(\mathrm{P}=0.07$ and $\mathrm{P}=0.077$, respectively; Fig. 5; Table III).

Pten methylation status. Among the $22 \mathrm{CpG}$ units of the Pten promoter in the thymus, CpG-11.12, CpG-23 and CpG-24.25 $(3 / 22,13.6 \%)$ demonstrated different methylation statuses in the control, tumor and non-tumor groups (Fig. 6; Table III). The methylation of CpG-11.12 in the tumor group was notably higher compared with in the non-tumor group $(\mathrm{P}=0.044)$; however, no statistical differences in methylation status were identified between the control and tumor/non-tumor groups. The methylation of CpG-23 in the tumor group 
Table II. Protein expression levels of TRP53, PTEN and MSH2 in four types of tissue.

\begin{tabular}{|c|c|c|c|c|c|c|c|c|c|c|c|c|}
\hline \multirow[b]{2}{*}{ Expression } & \multicolumn{3}{|c|}{ Thymus } & \multicolumn{3}{|c|}{ Liver } & \multicolumn{3}{|c|}{ Kidney } & \multicolumn{3}{|c|}{ Spleen } \\
\hline & $\mathrm{C}$ & $\mathrm{T}$ & NT & $\mathrm{C}$ & $\mathrm{T}$ & NT & C & $\mathrm{T}$ & NT & C & $\mathrm{T}$ & NT \\
\hline TRP35 & - & $\downarrow^{\mathrm{a}}$ & $\downarrow^{\mathrm{b}}$ & - & $\downarrow$ & $\uparrow$ & - & $\downarrow$ & $\uparrow$ & - & $\downarrow^{\mathrm{c}}$ & $\uparrow$ \\
\hline PTEN & - & $\downarrow^{\mathrm{d}}$ & $\uparrow$ & - & $\uparrow$ & $\uparrow$ & - & $\uparrow$ & $\uparrow$ & - & $\downarrow$ & $\uparrow$ \\
\hline MSH2 & - & $\uparrow$ & $\uparrow$ & - & $\uparrow^{\mathrm{e}}$ & $\uparrow$ & - & $\uparrow^{\mathrm{f}}$ & $\downarrow$ & - & $\uparrow^{\mathrm{g}}$ & $\uparrow$ \\
\hline
\end{tabular}

$\uparrow$, protein expression level is upregulated; $\downarrow$, protein expression level is downregulated. ${ }^{a} \mathrm{PC}=0.007 ;{ }^{b} \mathrm{PC}=0.031 ;{ }^{\mathrm{c}} \mathrm{P}_{\mathrm{C}}=0.009, \mathrm{P}_{\mathrm{NT}}=0.008$; ${ }^{d} \mathrm{P}_{\mathrm{C}}=0.027, \mathrm{P}_{\mathrm{NT}}=0.01 ;{ }^{\mathrm{e}} \mathrm{P}_{\mathrm{C}}<0.000, \mathrm{P}_{\mathrm{NT}}<0.000 ;{ }^{2} \mathrm{P}_{\mathrm{C}}=0.003, \mathrm{P}_{\mathrm{NT}}=0.002 ;{ }^{\mathrm{g}} \mathrm{P}_{\mathrm{C}}=0.012$. C, control; $\mathrm{T}$, tumor; NT, non-tumor; TRP53, transformation protein 53; PTEN, phosphatase and tensin homolog; MSH2, mutS homolog 2.
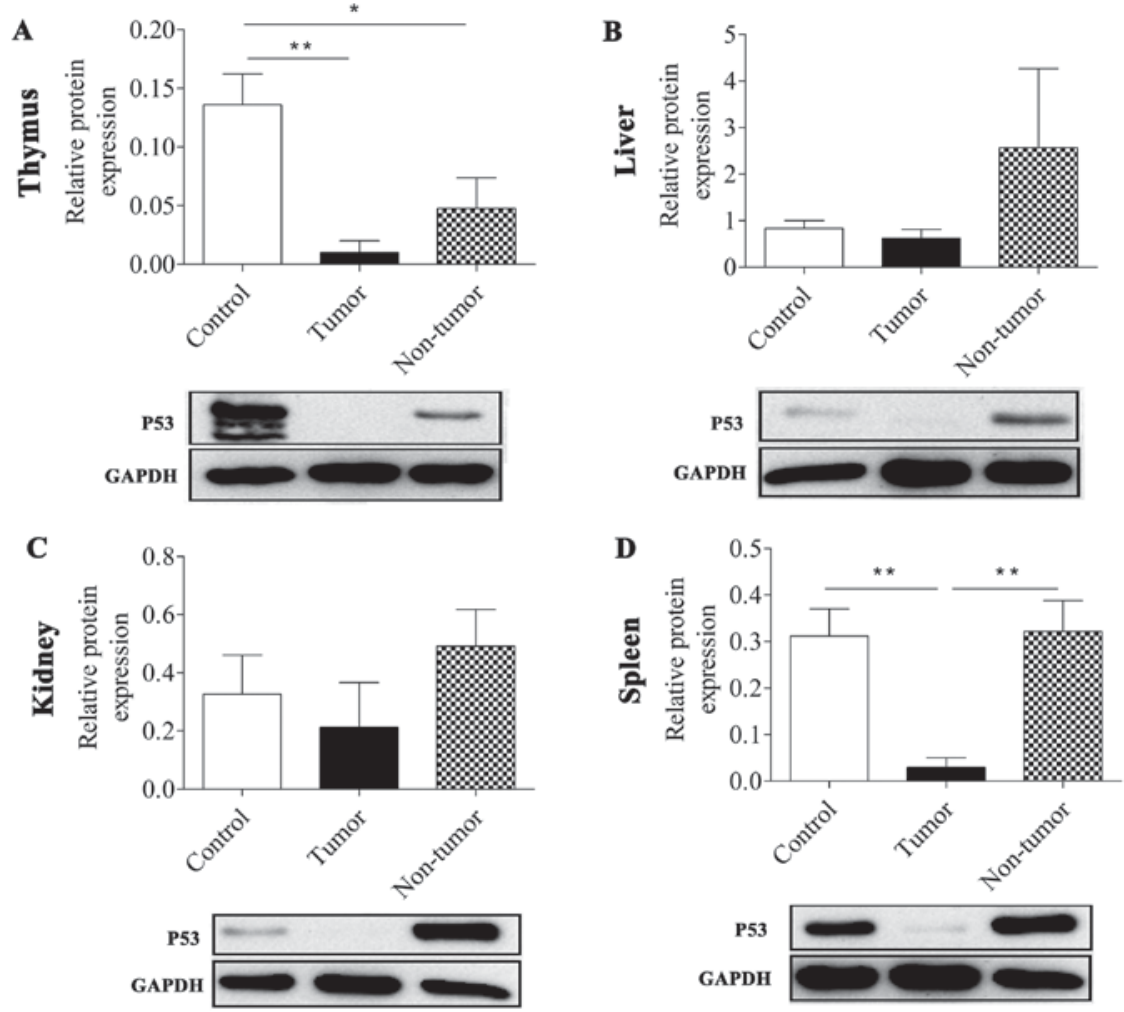

Figure 1. P53 protein expression levels in MNU-induced tissues, as determined by western blotting. P53 protein expression levels were assessed in (A) the thymus, (B) the liver, (C) the kidney and (D) the spleen. The control group was not treated with MNU, the tumor group was exposed to MNU and developed tumors, while the non-tumor group was exposed to MNU but did not develop tumors. ${ }^{*} \mathrm{P}<0.05$ and ${ }^{* *} \mathrm{P}<0.01$, with comparisons indicated by lines. P53, tumor protein 53; MNU, N-methyl-N-nitrosourea.

was significantly higher compared with in the control and non-tumor groups $(\mathrm{P}=0.003$ and $\mathrm{P}=0.007$, respectively); however, there was no statistical difference in methylation status between the non-tumor and control groups. The methylation of $\mathrm{CpG}-24.25$ in the tumor and non-tumor groups were significantly higher compared with in the control group $(\mathrm{P}=0.027$ and $\mathrm{P}=0.008$, respectively), whereas no statistical differences in methylation status were revealed between the tumor and non-tumor groups.

Msh2 methylation status. In the liver, CpG-10.11 (14.15), CpG-12 and CpG-21 (4/22, 18.2\%) in 22 CpG units of the $M s h 2$ promoter exhibited alterations in methylation status across the three groups. The methylation of CpG-10.11
(14.15) and CpG-21 in the non-tumor group was significantly increased compared with in the control group $(\mathrm{P}=0.036$ and $\mathrm{P}=0.028$, respectively). Conversely, the methylation of $\mathrm{CpG}-12$ in the non-tumor group was significantly lower compared with in the control group $(\mathrm{P}=0.048)$. No other differences were detected in these $\mathrm{CpG}$ sites. The methylation of CpG-20 in the tumor and non-tumor groups demonstrated a decreased tendency compared with in the control group $(\mathrm{P}=0.066$ and $\mathrm{P}=0.066$, respectively; Fig. 7; Table III).

In the kidney, two notable $\mathrm{CpG}$ units $(2 / 22,9.1 \%)$ of the $M s h 2$ promoter were captured. CpG-5 of the $M s h 2$ promoter in the non-tumor group demonstrated a significantly increased methylation status compared with in the control 

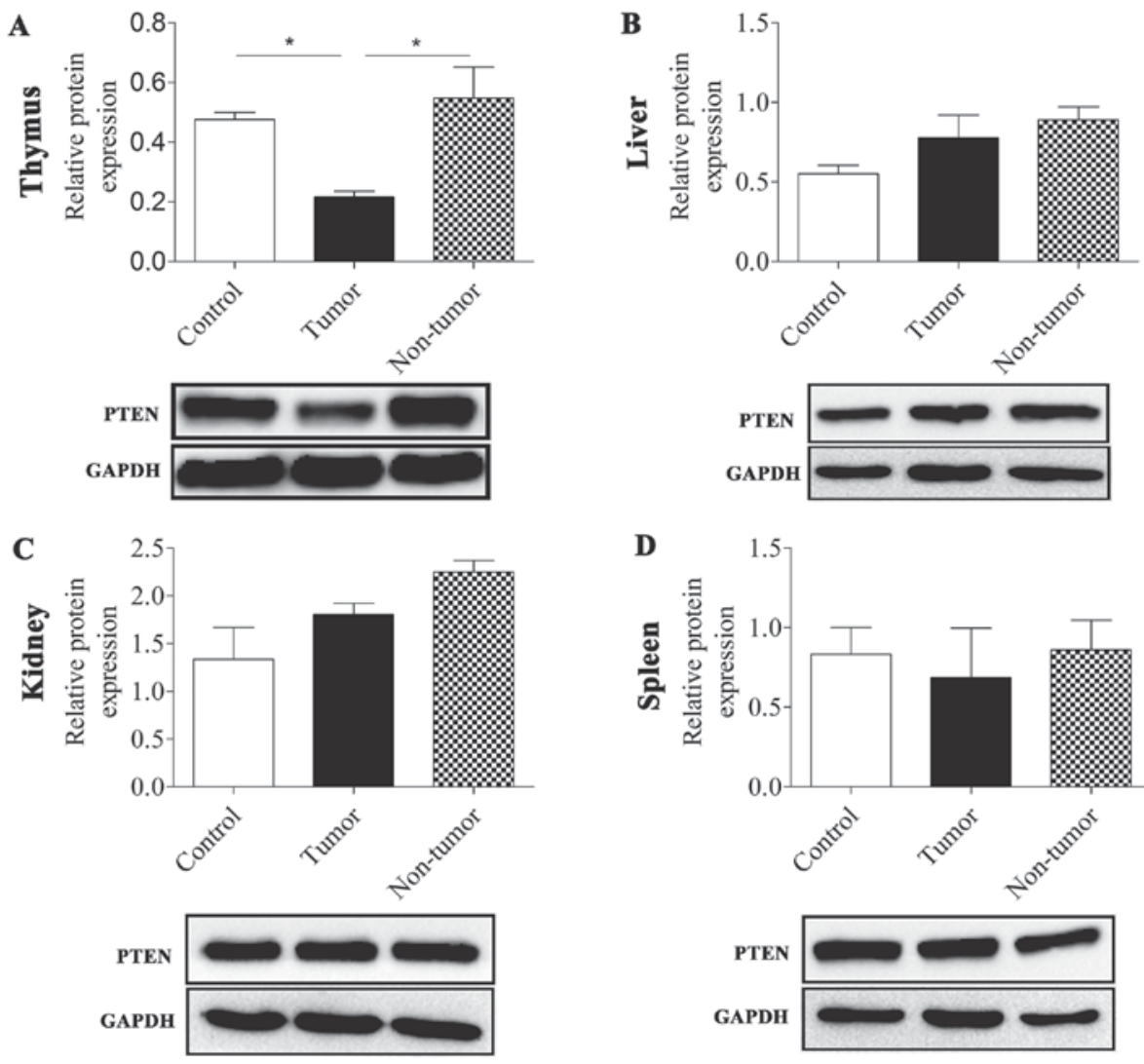

Figure 2. PTEN protein expression levels in MNU-induced tissues, as determined by western blotting. PTEN protein expression levels were assessed in (A) the thymus, (B) the liver, (C) the kidney and (D) the spleen. The control group was not treated with MNU, the tumor group was exposed to MNU and developed tumors, while the non-tumor group was exposed to MNU but did not develop tumors. ${ }^{\text {}} \mathrm{P}<0.05$, with comparisons indicated by lines. PTEN, phosphatase and tensin homolog; MNU, N-methyl-N-nitrosourea.
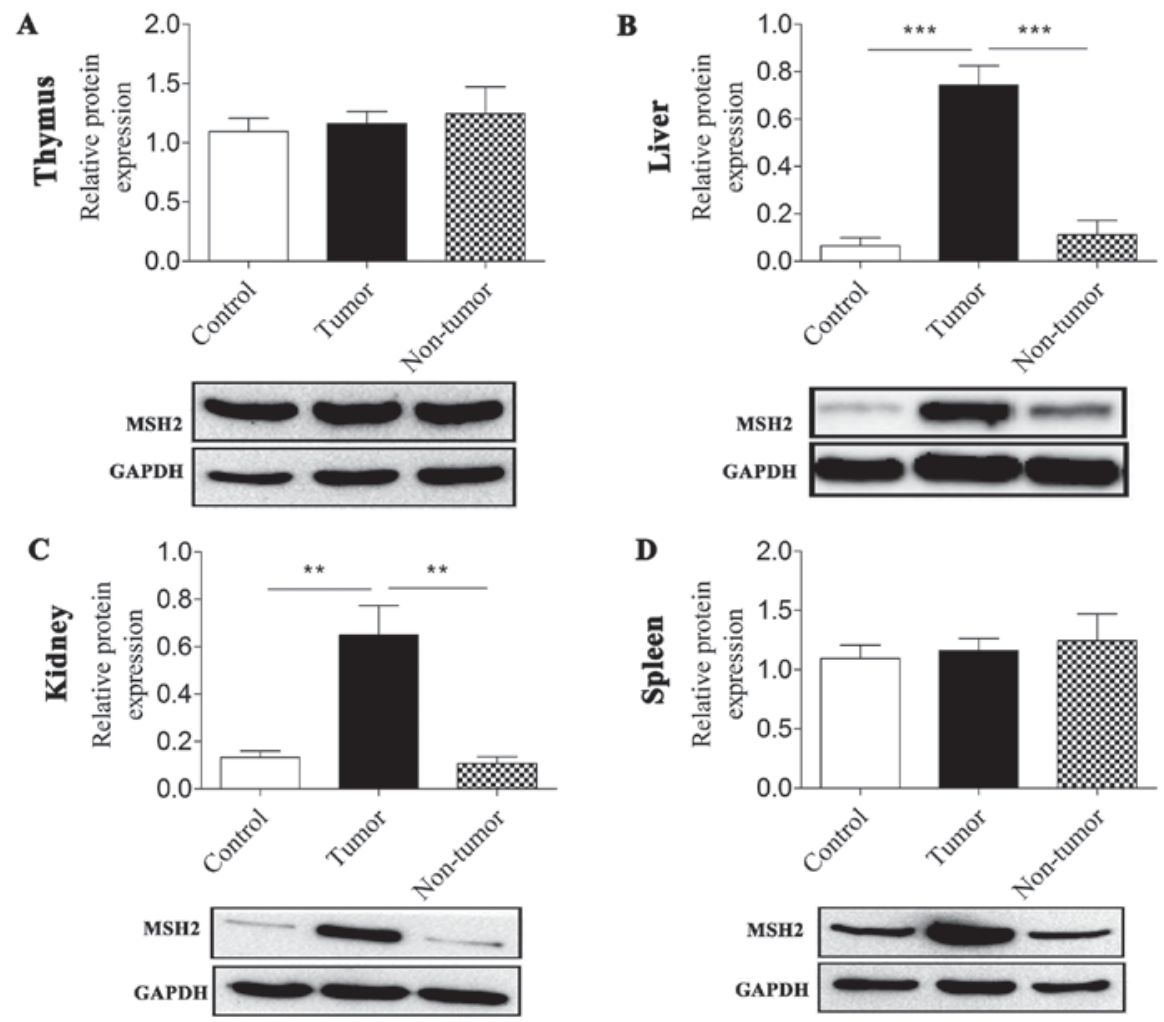

Figure 3. MSH2 protein expression levels in MNU-induced tissues. MSH2 protein expression levels were assessed in (A) the thymus, (B) the liver, (C) the kidney and (D) the spleen. The control group was not treated with MNU, the tumor group was exposed to MNU and developed tumors, while the non-tumor group was exposed to MNU but did not develop tumors. ${ }^{* *} \mathrm{P}<0.01$ and ${ }^{* * * *} \mathrm{P}<0.001$, with comparisons indicated by lines. MSH2, mutS homolog 2 ; MNU, $\mathrm{N}$-methyl-N-nitrosourea. 


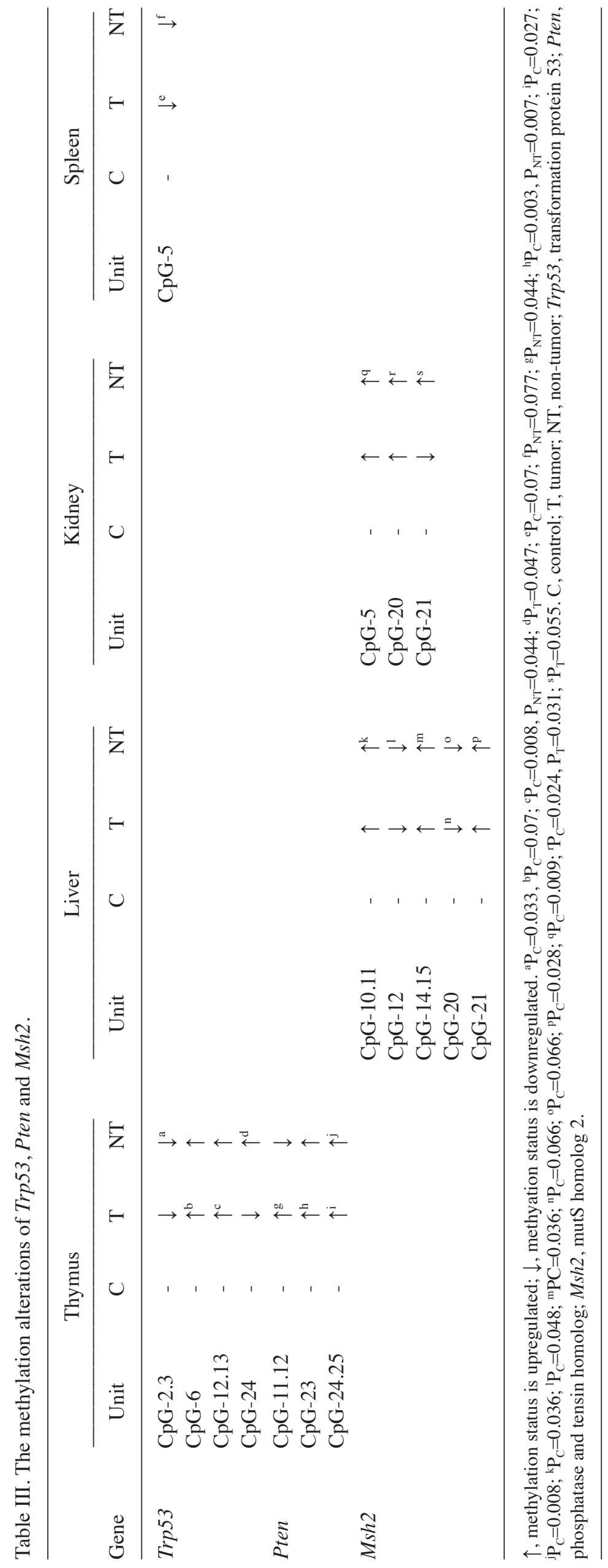



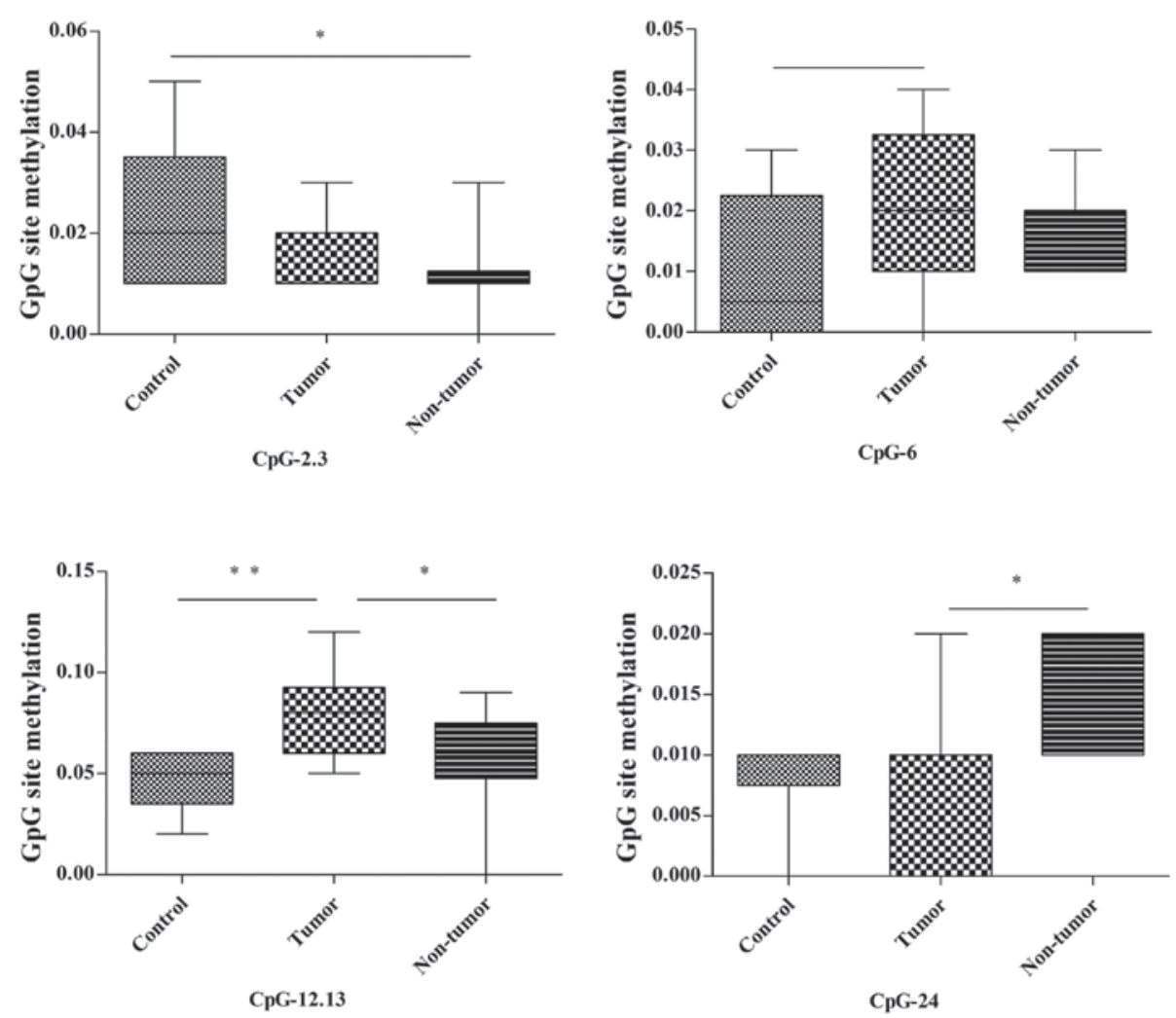

Figure 4. Methylation of CpG-2.3, CpG-12.13 and CpG-24 of the Trp53 promoter in the thymus. The control group was not treated with MNU, the tumor group was exposed to MNU and developed tumors, while the non-tumor group was exposed to MNU but did not develop tumors. ${ }^{*} \mathrm{P}<0.05$ and ${ }^{* *} \mathrm{P}<0.01$, with comparisons indicated by lines. CpG, 5'-C-phosphate-G-3'; Trp53, transformation protein 53; MNU, N-methyl-N-nitrosourea.

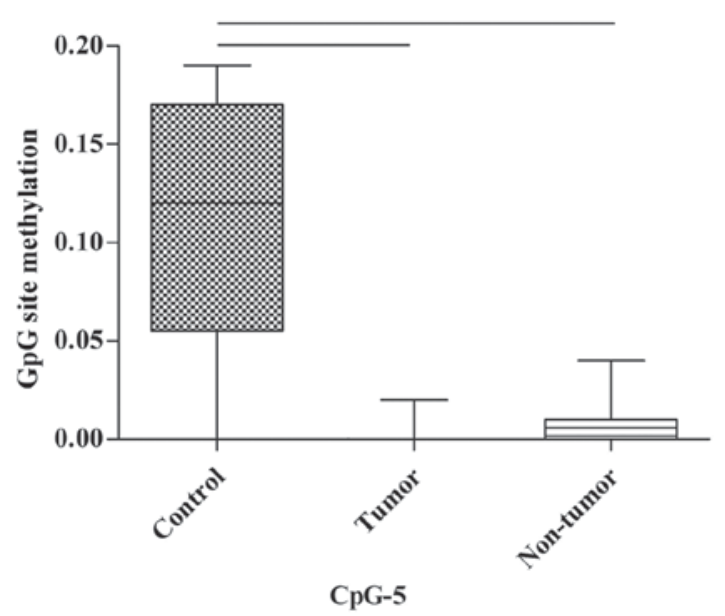

Figure 5. Methylation of CpG-5 of the $p 53$ promoter in the spleen. The control group was not treated with MNU, the tumor group was exposed to MNU and developed tumors, whereas the non-tumor group was exposed to MNU but did not develop tumors. CpG, 5'-C-phosphate-G-3'; p53, tumor protein P53.

group $(\mathrm{P}=0.009)$ and a slightly higher methylation tendency compared with in the tumor group $(\mathrm{P}=0.068)$. Similarly, the methylation of $\mathrm{CpG}-20$ in the non-tumor group was significantly higher compared with in the control and tumor groups $(\mathrm{P}=0.024$ and $\mathrm{P}=0.031$, respectively). No significant differences were identified in the $\mathrm{CpG}-5$ and $\mathrm{CpG}-20$ units. Furthermore, the methylation of $\mathrm{CpG}-21$ in the non-tumor group tended to be higher compared with in the tumor group $(\mathrm{P}=0.055$; Fig. 8; Table III).
In the spleen, none of the $22 \mathrm{CpG}$ sites demonstrated significant changes of methylation status between the various groups.

\section{Discussion}

Although numerous genes encode tumor suppressors, in general, each is dedicated to specific defense pathways (19). Therefore, these genes may act during various periods of tumorigenesis, and may provide a more robust contribution when they are simultaneously activated. For instance, $M s h 2$ and $T r p 53$ promote genome stability. However, Msh2 contributed to the suppression of spontaneous microsatellite instability (MSI) by directly eliminating altered sequences at the nucleotide level, but had no effect on radiation-induced MSI (19). Conversely, TRP53 suppressed radiation-induced MSI by removing damaged cells, but did not affect spontaneous MSI in a previous study (19). Thus, the present study inferred that it is essential that groups of tumor suppressor genes are examined concomitantly.

In the present study, the protein expression level of TRP53 decreased in the thymus in the tumor and non-tumor groups, indicating that MNU induction impaired TRP53 expression. The marked reduction of TRP53 protein expression in the tumor group may suggest that the occurrence of thymic lymphoma is associated with TRP53 deficiency, which is consistent with previous studies that reducing TRP53 promoted the development of thymic lymphoma (20). In the spleen, the TRP53 expression levels in the tumor group were markedly lower compared with in the control and non-tumor groups. This suggested that there may be 

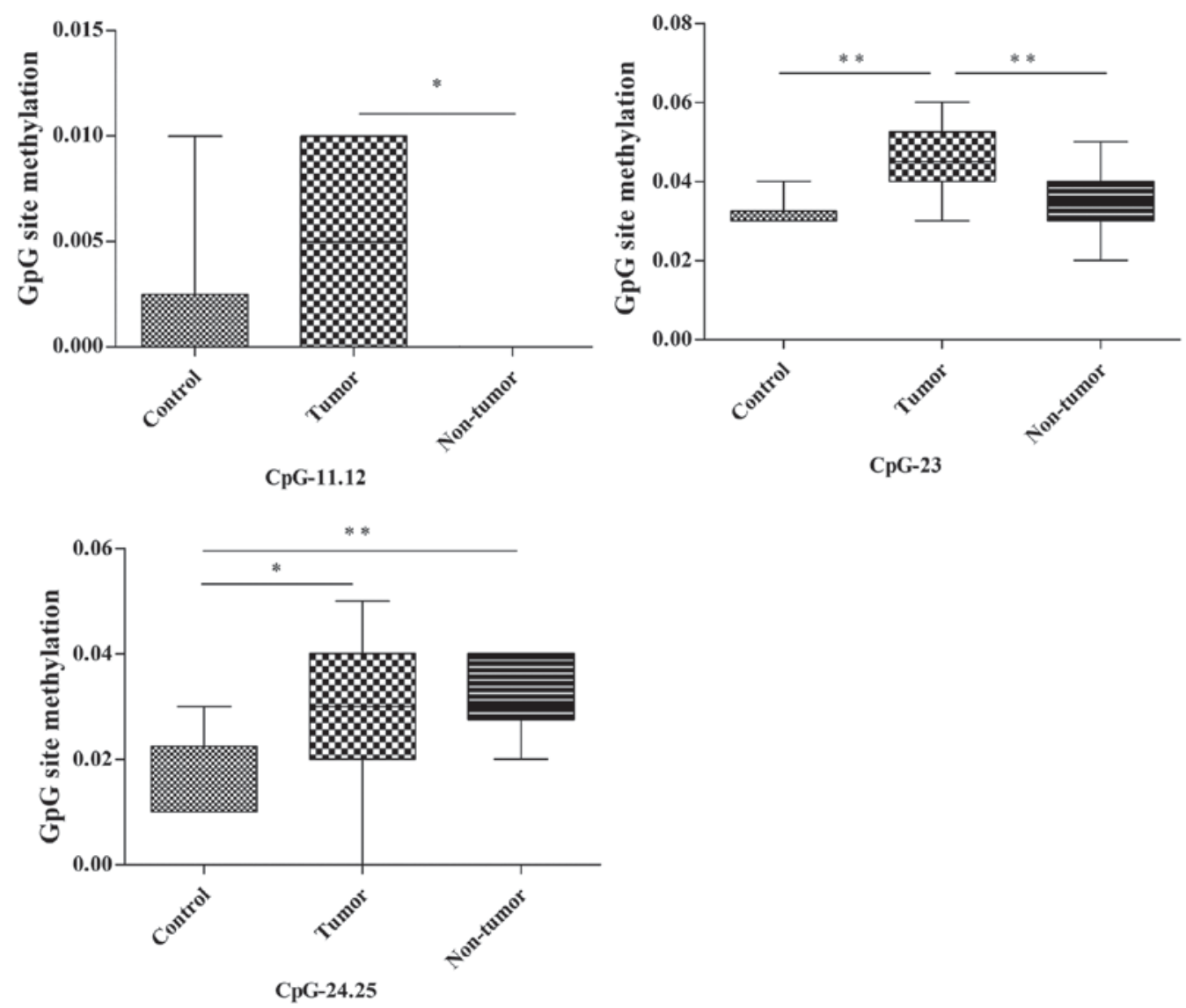

Figure 6. Methylation of CpG-11.12, CpG-23 and CpG-24.25 of the Pten promoter in the thymus. The control group was not treated with MNU, the tumor group was exposed to MNU and developed tumors, while the non-tumor group was exposed to MNU but did not develop tumors. " $\mathrm{P}<0.05$ and ${ }^{* *} \mathrm{P}<0.01$, with comparisons indicated by lines. CpG, 5'-C-phosphate-G-3'; Pten, phosphatase and tensin homolog; MNU, N-methyl-N-nitrosourea..

an association between TRP53 deficiency and metastasis of thymic lymphoma to the spleen.

In the thymus, the downregulation of PTEN in the thymic lymphoma group but not in the control and non-tumor groups suggested that PTEN loss may be an aggravating factor in tumor development. This was supported by a previous study, which revealed that PTEN deficiency serves an important function in the oncogenesis of $\mathrm{T}$ cell lymphoma (21), and Pten-knockout mice will develop T cell lymphoma uniformly within 10-16 weeks (22-25). No significant difference in the expression levels of PTEN in the control, tumor and non-tumor groups of the liver, kidney and spleen were identified, indicating that there was no association between PTEN expression level and thymic lymphoma metastasis.

The result that there was no significant difference in MSH2 expression levels in the control, tumor and non-tumor thymus groups suggested that MSH2 does not serve a major function in the formation of thymic lymphoma. However, the expression levels of MSH2 in the liver, kidney and spleen demonstrated a positive association between MSH2 overexpression and the metastasis of thymic lymphoma.

According to the aforementioned results, the present study hypothesized that mice that revealed a reduction in P53 and PTEN expression levels may be prone to thymic lymphoma development, that the reduced expression level of TRP53 may promote spleen metastasis of thymic lymphoma and that the upregulation of MSH2 may be associated with metastasis of thymic lymphoma to the liver, kidney and spleen. However, further studies are required to confirm these hypotheses.

MNU is an alkylating agent that induces extensive methylation of DNA and hypermethylation in the promoter regions, which may consequently induce the silencing of numerous tumor suppressor genes. Accordingly, the present study hypothesized that aberrant methylations may epigenetically repress gene expression and promote the formation of thymic lymphoma. However, the methylation state is not often detectable in tissue samples (11) and no study has analyzed the DNA methylation of P53, Pten and Msh2 in MNU-induced thymic lymphoma. The present study investigated the methylation status of Trp53, Pten and $M s h 2$ in four types of MNU-induced tissues.

The reduction of P53 expression levels in thymic lymphoma may be associated with the hypermethylation of $\mathrm{CpG}-12.13$ and the hypermethylation tendency of CpG-6 in the Trp53 promoter, and P53 expression in the thymic lymphoma was revealed to be lower compared with that of the non-tumor group. This may partially be due to the hypermethylation of CpG-12.13.

The downregulation of PTEN in thymic lymphoma may be associated with the hypermethylation of CpG-23 in Pten. The lower PTEN expression levels observed in thymic lymphoma compared with in the non-tumor group may be associated with the hypermethylation of $\mathrm{CpG}-23$ and $\mathrm{CpG}-11.12$ compared with the non-tumor group. CpG-24.25, which was hypermethylated in the tumor and non-tumor thymus tissues, may 


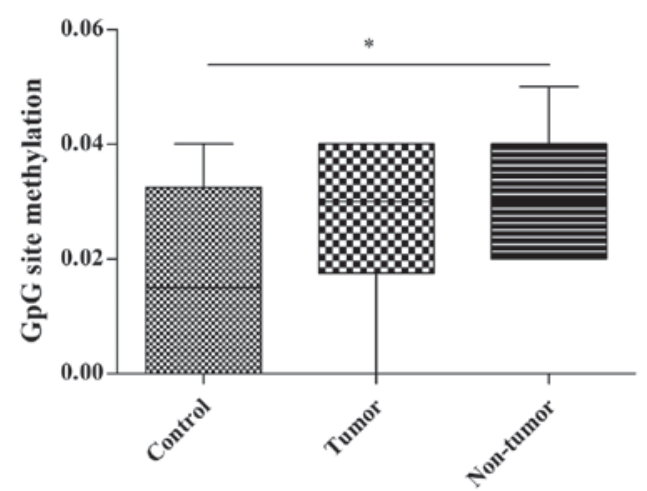

CpG-10.11(14.15)

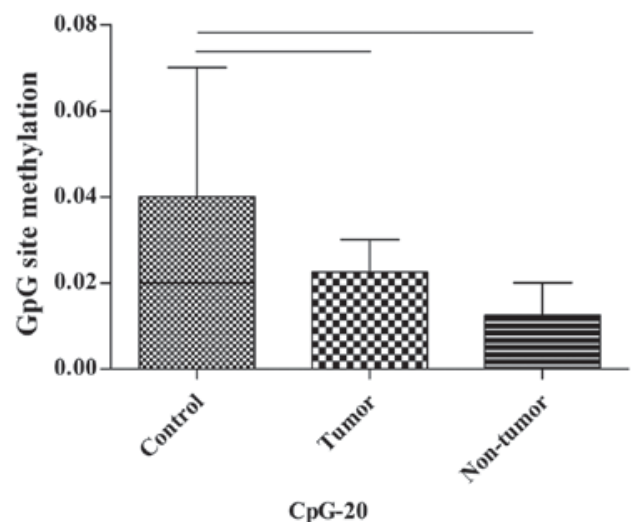

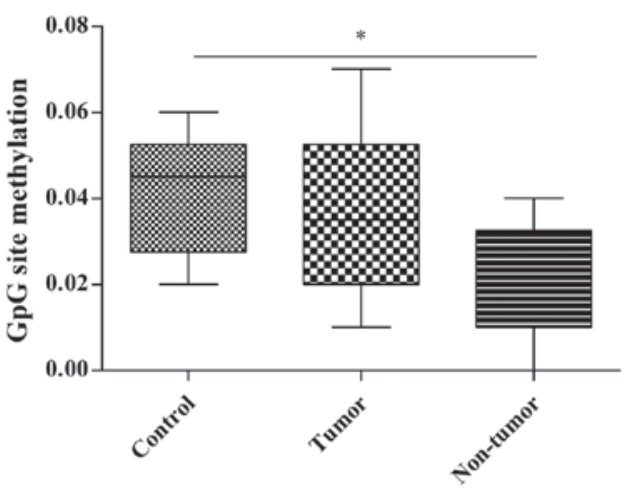

CpG-12

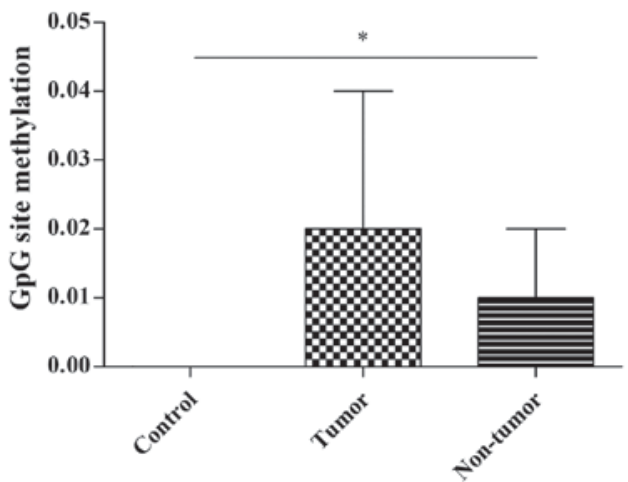

CpG-21

Figure 7. Methylation of CpG-10.11 (14.15), CpG-12, CpG-20 and CpG-21 of the Msh2 promoter in the liver. The control group was not treated with MNU, the tumor group was exposed to MNU and developed tumors, while the non-tumor group was exposed to MNU but did not develop tumors. "P<0.05, with comparisons indicated by lines. CpG, 5'-C-phosphate-G-3'; Msh2, mutS homolog 2; MNU, N-methyl-N-nitrosourea.
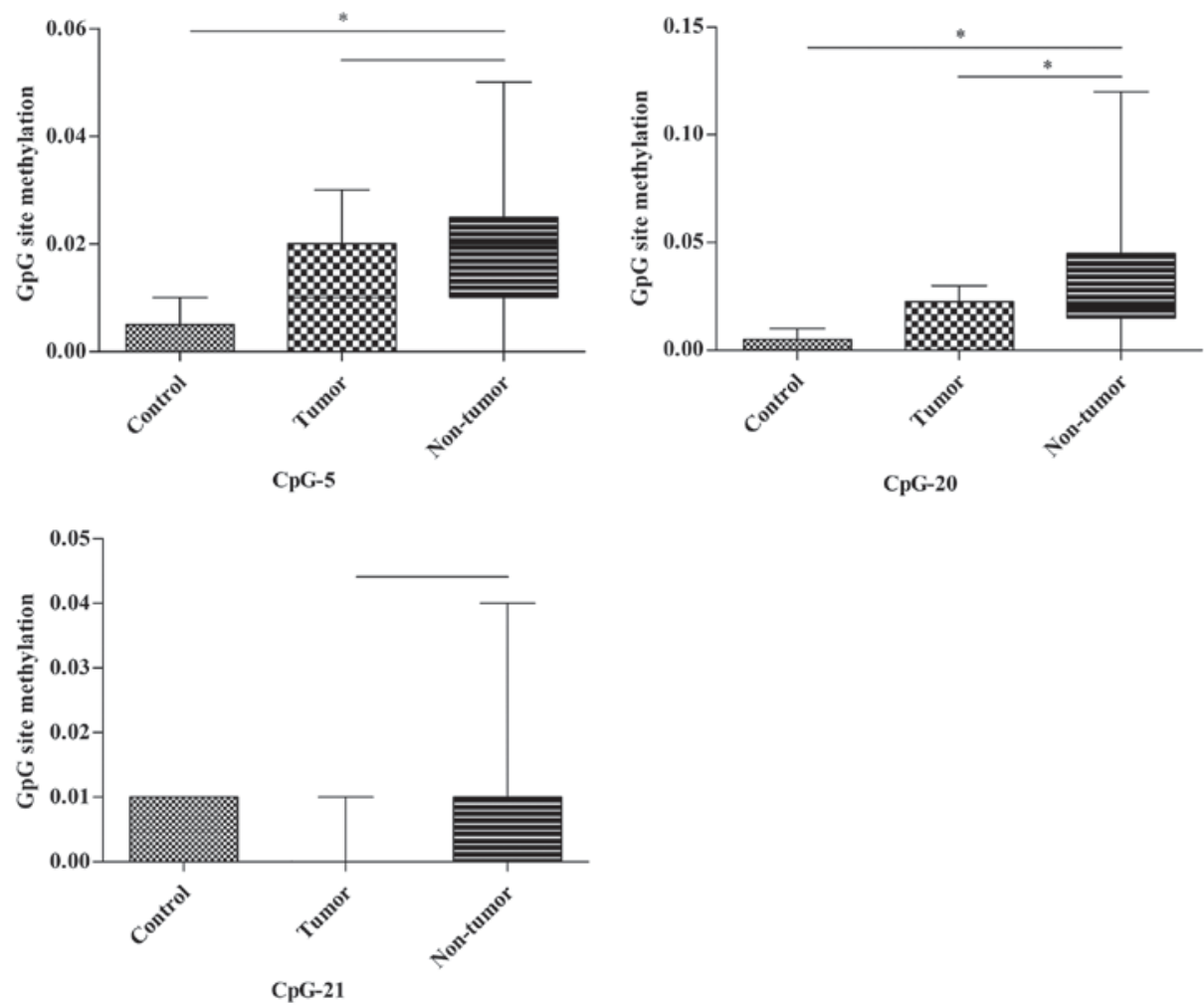

Figure 8. Methylation of CpG-5, CpG-20 and CpG-21 of the $M s h 2$ promoter in the kidney. The control group was not treated with MNU, the tumor group was exposed to MNU and developed tumors, while the non-tumor group was exposed to MNU but did not develop tumors. "P $<0.05$, with comparisons indicated by lines. CpG, 5'-C-phosphate-G-3'; Msh2, mutS homolog 2; MNU, N-methyl-N-nitrosourea. 
be associated with MNU treatment but not PTEN expression levels.

In the liver, the hypermethylation of CpG-10.11 $(14,15)$, CpG-21 and the hypomethylation of CpG-12 in Msh2, which were all uniquely detected in non-tumor groups, may be associated with tumor-free status following MNU induction. Similarly, in the kidney, the hypermethylation of CpG-5, CpG-20 may be associated with tumor-free status following MNU induction. However, all the aforementioned assumptions, which are based on the results of DNA methylation analysis, require further confirmation.

The present study described the changes in the expression levels of PTEN, TRP53 and MSH2 concomitantly in MNU-induced thymic lymphoma, and from the results it was inferred that tumor suppressor genes may be involved in various stages of tumor development. The present study also investigated the promoter methylation profile of each gene and deduced that the methylation status of certain $\mathrm{CpG}$ sites was involved in the regulation of gene expression. Furthermore, Song et al (11) revealed that hypermethylation of $8 \mathrm{CpG}$ sites in the 16 promoter may reduce its expression level in the thymic lymphoma following irradiation; a similar result to the present study. Additionally, in the Msh2 promoter, the $\mathrm{CpG}$ sites in which methylation status changed in the non-tumor group specifically may be potential markers of tumor-free status. These results help to further understanding of the development of lymphoma. Nevertheless, the underlying molecular mechanisms of Pten, Trp53 and Msh2 cooperation during tumorigenesis remain unclear. Further studies are required to investigate the function of these genes and epigenetic regulation in mutagen-induced cancer.

\section{References}

1. Han SW, Lee HJ, Bae JM, Cho NY, Lee KH, Kim TY, Oh DY, Im SA, Bang YJ, Jeong SY, et al: Methylation and microsatellite status and recurrence following adjuvant FOLFOX in colorectal cancer. Int J Cancer 132: 2209-2216, 2013.

2. Salmena L, Carracedo A and Pandolfi PP: Tenets of PTEN tumor suppression. Cell 133: 403-414, 2008.

3. Li T, Kon N, Jiang L, Tan M, Ludwig T, Zhao Y, Baer R and $\mathrm{Gu}$ W: Tumor suppression in the absence of p53-mediated cell-cycle arrest, apoptosis, and senescence. Cell 149: 1269-1283, 2012.

4. Vazquez F and Devreotes P: Regulation of PTEN function as a PIP3 gatekeeper through membrane interaction. Cell Cycle 5: 1523-1527, 2006.

5. Vousden KH and Prives C: P53 and prognosis: New insights and further complexity. Cell 120: 7-10, 2005.

6. Soria JC, Lee HY, Lee JI, Wang L, Issa JP, Kemp BL, Liu DD, Kurie JM, Mao L and Khuri FR: Lack of PTEN expression in non-small cell lung cancer could be related to promoter methylation. Clin Cancer Res 8: 1178-1184, 2002.

7. Jesionek-Kupnicka D, Szybka M, Malachowska B, Fendler W, Potemski P, Piaskowski S, Jaskolski D, Papierz W, Skowronski W, Och W, et al: TP53 promoter methylation in primary glioblastoma: Relationship with TP53 mRNA and protein expression and mutation status. Dna Cell BioL 33: 217-226, 2014.
8. Zeng X and Kinsella TJ: A novel role for DNA mismatch repair and the autophagic processing of chemotherapy drugs in human tumor cells. Autophagy 3: 368-370, 2007.

9. Harfe BD and Jinks-Robertson S: Sequence composition and context effects on the generation and repair of frameshift intermediates in mononucleotide runs in Saccharomyces cerevisiae. Genetics 156: 571-578, 2000.

10. Campbell MR, Wang Y, Andrew SE and Liu Y: Msh2 deficiency leads to chromosomal abnormalities, centrosome amplification, and telomere capping defect. Oncogene 25: 2531-2536, 2006.

11. Song W, Liu Y, Liu Y, Zhang C, Yuan B, Zhang L and Sun S: Increased P16 DNA methylation in mouse thymic lymphoma induced by irradiation. PLoS One 9: e93850, 2014.

12. Douet V, Heller MB and Le Saux O: DNA methylation and Sp1 binding determine the tissue-specific transcriptional activity of the mouse Abcc6 promoter. Biochem Biophys Res Commun 354: 66-71, 2007.

13. Suzuki MM and Bird A: DNA methylation landscapes: Provocative insights from epigenomics. Nat Rev Genet 9: 465-476, 2008.

14. Nishida N and Kudo M: Alteration of epigenetic profile in human hepatocellular carcinoma and its clinical implications. Liver Cancer 3: 417-427, 2014.

15. Singla N and Dhawan DK: N-methyl N-nitrosourea induced functional and structural alterations in mice brain-role of curcumin. Neurotox Res 22: 115-126, 2012.

16. Chang YC, Hsu JD, Lin WL, Lee YJ and Wang CJ: High incidence of acute promyelocytic leukemia specifically induced by N-nitroso-N-methylurea (NMU) in Sprague-Dawley rats. Arch Toxicol 86: 315-327, 2012.

17. Huo X, Zhang S, Li Z, Gao J, Wang C, Li C, Guo M, Du X and Chen Z: Analysis of the relationship between microsatellite instability and thymic lymphoma induced by $\mathrm{N}$-methyl-N-nitrosourea in C57BL/6J mice. Mutat Res 771: 21-28, 2015.

18. Coolen MW, Statham AL, Gardiner-Garden M and Clark SJ: Genomic profiling of $\mathrm{CpG}$ methylation and allelic specificity using quantitative high-throughput mass spectrometry: Critical evaluation and improvements. Nucleic Acids Res 35: e119, 2007.

19. Otozai S, Ishikawa-Fujiwara T, Oda S, Kamei Y, Ryo H, Sato A, Nomura T, Mitani $\mathrm{H}$, Tsujimura $\mathrm{T}$, Inohara $\mathrm{H}$ and Todo $\mathrm{T}$ : p53-Dependent suppression of genome instability in germ cells. Mutat Res 760: 24-32, 2014.

20. Kamimura K, Ohi H, Kubota T, Okazuka K, Yoshikai Y, Wakabayashi Y, Aoyagi Y, Mishima Y and Kominami R: Haploinsufficiency of Bcll1b for suppression of lymphomagenesis and thymocyte development. Biochem Biophys Res Commun 355: 538-542, 2007

21. Guo W, Schubbert S, Chen JY, Valamehr B, Mosessian S, Shi H, Dang NH, Garcia C, Theodoro MF, Varella-Garcia M and Wu H: Suppression of leukemia development caused by PTEN loss. Proc Natl Acad Sci USA 108: 1409-1414, 2011.

22. Buckler JL, Walsh PT, Porrett PM, Choi Y and Turka LA: Cutting edge: $T$ cell requirement for $\mathrm{CD} 28$ costimulation is due to negative regulation of TCR signals by PTEN. J Immunol 177: 4262-4266, 2006.

23. Hagenbeek TJ and Spits H: T-cell lymphomas in T-cell-specific Pten-deficient mice originate in the thymus. Leukemia 22: 608-619, 2008.

24. Suzuki A, Yamaguchi MT, Ohteki T, Sasaki T, Kaisho T, Kimura Y, Yoshida R, Wakeham A, Higuchi T, Fukumoto M, et al: T cell-specific loss of Pten leads to defects in central and peripheral tolerance. Immunity 14: 523-534, 2001.

25. Xue L, Nolla H, Suzuki A, Mak TW and Winoto A: Normal development is an integral part of tumorigenesis in T cell-specific PTEN-deficient mice. Proc Natl Acad Sci USA 105: 2022-2027, 2008. 